\title{
Quasi-absolute neutron-induced fission cross section of ${ }^{243} \mathrm{Am}$
}

\author{
M. Aïche ${ }^{1, \text { a }}$, G. Kessedjian ${ }^{1}$, G. Barreau ${ }^{1}$, B. Jurado ${ }^{1}$, A. Bidaud ${ }^{1}$, S. Czajkowski ${ }^{1}$, D. Dassié ${ }^{1}$, B. Haas ${ }^{1}$, L. Mathieu ${ }^{1}$, \\ L. Tassan-Got ${ }^{2}$, J. Wilson ${ }^{2}$, F.-J. Hambsch ${ }^{3}$, S. Oberstedt ${ }^{3}$, I. AlMahamid ${ }^{4}$, J. Floyd ${ }^{5}$, W. Lukens ${ }^{5}$, and D. Shuh ${ }^{5}$ \\ 1 CENBG, Université de Bordeaux 1, 33175 Gradignan Cedex, France \\ 2 IPN Orsay, Université Paris-Sud, 91406 Orsay, France \\ 3 EC-JRC-IRMM, 2440 Geel, Belgium \\ 4 Laboratory of Inorganic and Nuclear Chemistry, NY 12201, USA \\ 5 LBNL, CA 94720, USA
}

\begin{abstract}
The existing evaluations of the ${ }^{243}$ Am neutron-induced fission cross section have been questioned by recent studies performed at the GNEISS facility. In the neutron energy range from 1 to $6 \mathrm{MeV}$ the GNEISS data present deviations of more than $15 \%$ with respect to the evaluations. In order to solve this problem, we have performed a first quasi-absolute measurement of this fission cross section from the fission threshold up to the onset of secondchance fission. To achieve this goal we have used the neutron-proton scattering cross section as reference reaction to determine the incident neutron flux. This cross section is known with a precision better than $1 \%$ for a wide range of neutron energies ( $1 \mathrm{meV}$ to $20 \mathrm{MeV})$.
\end{abstract}

\section{Introduction}

${ }^{243} \mathrm{Am}$ is present in a rather important amount in the waste generated by current PWR nuclear reactors. Nowadays, ${ }^{241} \mathrm{Am}$ and ${ }^{243} \mathrm{Am}$ are the only isotopes that can be fully separated and extracted from spent fuel rods. Therefore, they represent the only nuclei for which fast neutron incineration could be seriously considered in a relatively near future. A reliable design of incineration reactors requires the precise knowledge (around $5 \%$ of accuracy) of the neutron-induced fission cross section of ${ }^{243} \mathrm{Am}$ in a fast neutron spectrum. However, in the 1 to $6 \mathrm{MeV}$ neutron-energy range, the existing data indicate systematic and significant discrepancies and could be segregated into two distinct groups. The experimental data of the first group set by Behrens et al. ref. [1] and Goverdovsky et al. ref. [2] give systematically higher fission cross section than the second group set by Knitter et al. ref. [3] and Seeger et al. ref. [4]. The most recent data from Laptev et al. in ref. [5] lie in the higher-cross section group which is more than $15 \%$ above evaluations and values of the second group. Actually, this discrepancy seems to be related to a normalisation problem as it has been discussed recently by Talou et al. in ref. [6].

In order to solve this controversy, we have performed for the first time a quasi-absolute measurement of the ${ }^{243} \mathrm{Am}$ neutron-induced fission cross section. Most of the fission data on ${ }^{243} \mathrm{Am}$ have been measured in reference to the ${ }^{235} \mathrm{U}$ neutron-induced fission cross section. In our case we have used the neutron-proton scattering cross section as a reference reaction to determine the incident neutron flux.

While for the ${ }^{235} \mathrm{U}$ fission cross section, the dispersion between available data is about $3 \%$, the neutron-proton scattering cross section is known with a precision better than $1 \%$ for a wide range of neutron energies ( $1 \mathrm{meV}$ to $20 \mathrm{MeV}$, refs. $[7,8])$. This high precision explains why we can call our measurement "quasi-absolute".

\footnotetext{
${ }^{a}$ Presenting author, e-mail: aiche@cenbg.in2p3.fr
}

\section{Experiment}

Cross-section measurements of ${ }^{243} \mathrm{Am}(\mathrm{n}, \mathrm{f})$ were performed at the 7MV Van-de-Graaff-driven neutron source facility of the IRMM in Belgium and more recently at the $4 \mathrm{MV}$ accelerator facility (AIFIRA) at CEN Bordeaux. The fast neutron flux over the neutron energy range $1 \mathrm{MeV}$ to $8 \mathrm{MeV}$ was produced by use of the $\mathrm{T}(\mathrm{p}, \mathrm{n})^{3} \mathrm{He}$ reaction up to $4 \mathrm{MeV}$ and the $\mathrm{D}(\mathrm{d}, \mathrm{n})^{3} \mathrm{He}$ reaction from 4 to $8 \mathrm{MeV}$.

Back-to-back deposits of two ${ }^{243} \mathrm{Am}$ targets each of about $550 \mu \mathrm{g} / \mathrm{cm}^{2}$ thickness were placed at a distance of $39 \mathrm{~mm}$ from the neutron source and at 0 degree with respect to the incidentneutron beam. Two sets of photovoltaic cells in a very compact geometry were placed at $5 \mathrm{~mm}$ on both sides of the Am targets. Each photovoltaic cell of rectangular shape $\left(20 \times 40 \mathrm{~mm}^{2}\right)$ consists of a monocristalline silicon structure covered with a comb-like conducting grid. These photo-voltaic cells operate without any applied bias leading to a very small depletion depth $(<0.2 \mu \mathrm{m})$, consequently light particles (e, p, $\alpha)$ loose only small fraction of their energy in the depletion zone. Regarding the detection of the fission-fragments, the photovoltaic cells present the advantage of having an intrinsic efficiency of 95\% and of being almost insensitive to the neutron beam and the alpha activity of the targets. The geometrical efficiency for detecting the fission fragments was approximately $70 \%$.

A polypropylene (PP) foil $\left(\left(\mathrm{C}_{3} \mathrm{H}_{6}\right)_{n}\right)$ was placed at $45 \mathrm{~mm}$ from the ${ }^{243} \mathrm{Am}$ targets and $80 \mathrm{~mm}$ from the neutron production source. This foil placed at 0 degree with respect to the incident-neutron beam served as a radiator to convert quasimonoenergetic neutrons into protons. Its thickness varied from 10 to $50 \mu \mathrm{m}$ depending on the neutron energy. Protons recoiling from neutron scattering in the PP foil were detected by means of $\Delta \mathrm{E}$-E telescope located at $75 \mathrm{~mm}$ from the foil. The ensemble PP-telescope formed our neutron flux detector. Its collimation aperture subtended a solid angle of $\sim 0.0075$ $10^{-2}$ of $4 \pi$ sr from the PP foil. Thus, the scattered protons were tightly collimated and only protons that moved near to the 

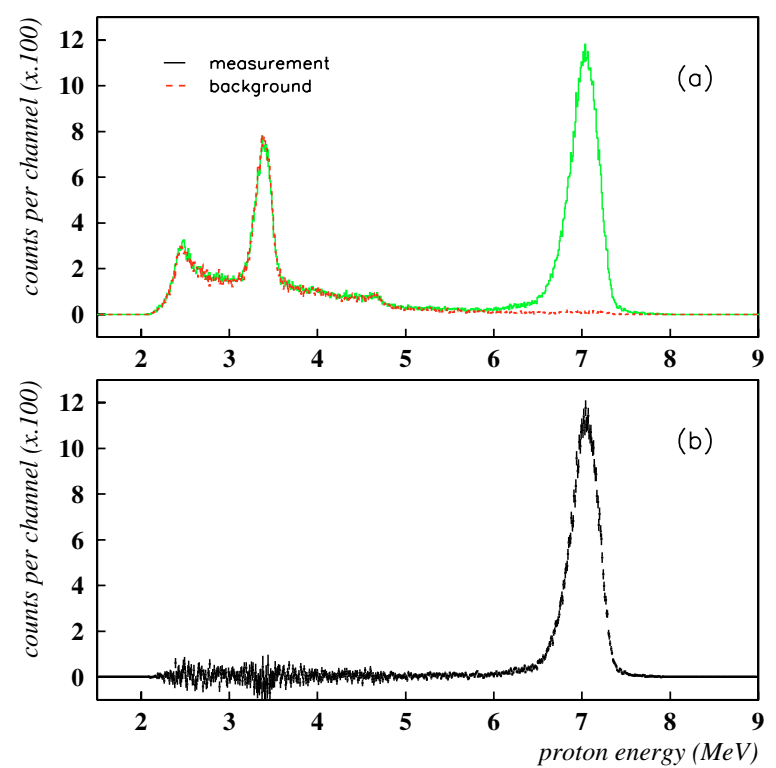

Fig. 1. Number of detected protons as a function of the total kinetic energy $(\Delta \mathrm{E}+\mathrm{E})$. (a) The standard measurement spectrum shows a pronounced proton peak around $7 \mathrm{MeV}$ if compared to the superimposed background spectrum. (b) The result after background subtraction. The latter shows the proton peak associated to the incident neutron flux.

incident neutron direction and therefore had nearly the same energy as the source neutrons, were detected.

The main concern of this experiment was the background generated by neutrons scattered from counter materials or other objects close to the Telescope detector. In addition, charged particles originating from the direct interaction of incoming neutrons with the $\Delta \mathrm{E}-\mathrm{E}$ Silicon were also detected by the Telescope. A measurement of the recoiling proton spectrum at each energy consisted of a standard and a background separate runs. For the standard measurement, the PP radiator was facing the Telescope. For the background measurement, the recoiling protons were stopped in a tantalum screen placed between the PP foil and the Telescope. The tantalum thickness was adequate to stop the highest energy protons in this experiment. A neutron monitor placed at 0 degree with respect to the incident-neutrons beam at $4.21 \mathrm{~m}$ from the neutron source assured the normalisation of the standard and background measurements.

In the following we will concentrate in the determination of the incident neutron flux from the protons scattered in the PP foil. The number of detected recoiling protons as a function of the total kinetic energy before and after background subtraction is shown in figure 1 .

At high neutron energy ( $>4.5 \mathrm{MeV}$ ) the background is mainly due to the direct reaction of the incident neutrons with the silicon detector ${ }^{29} \mathrm{Si}(\mathrm{n}, \alpha)^{26} \mathrm{Mg}$ since the alpha particles have the same energy as the scattered protons. Nevertheless, the spectrum that results from the background subtraction presents only one peak corresponding to the protons produced by the interaction of the quasi-monoenergetic incident neutrons with the PP foil. Monte Carlo simulations of neutrons passing the experimental setup have been performed in order to determine the average energy of the neutrons hitting the
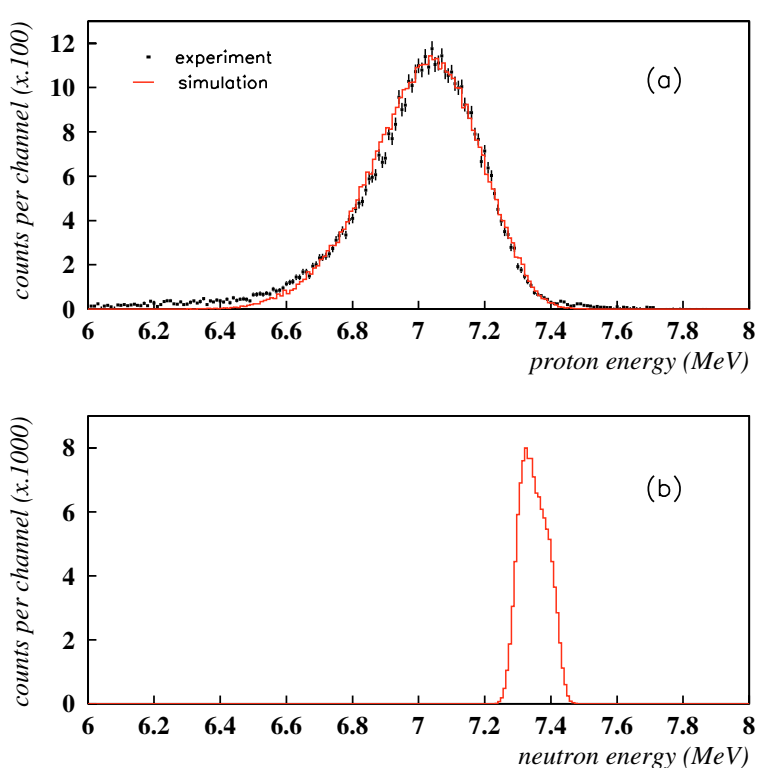

Fig. 2. (a) Experimental and simulated spectra of recoil protons as a function of total kinetic energy. (b) Simulated neutron spectrum as a function of kinetic energy.

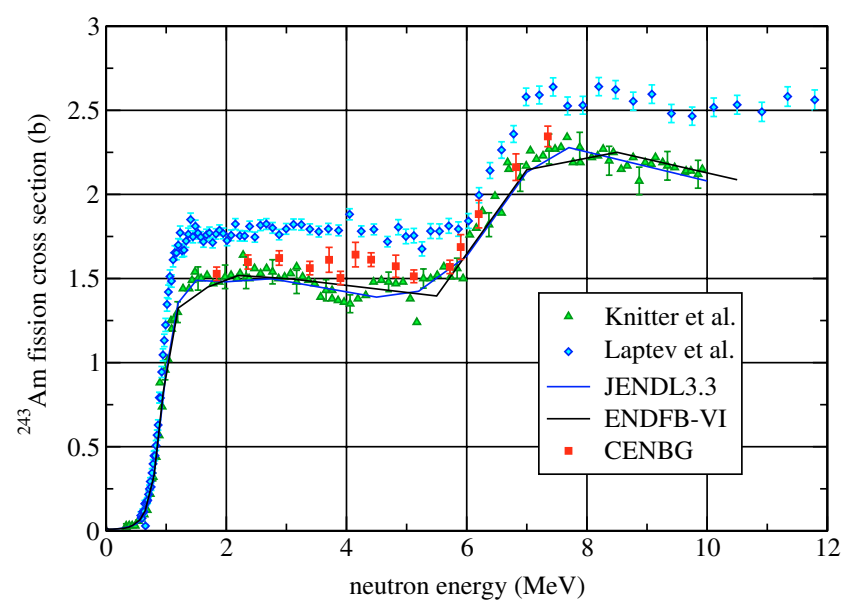

Fig. 3. Preliminary results for the ${ }^{243} \mathrm{Am}$ neutron-induced fission cross section of the present work are compared to international evaluations and experimental data.

${ }^{243} \mathrm{Am}$ targets and provide information about the features of the neutrons flux.

In a first step, the simulation takes into account the angular distributions of the neutrons emerging from the neutron source, the energy resolution effects of the incident beam and the energy loss of the charged particles in the neutron source. In a second step, neutrons emitted in the forward direction interact with the PP foil and generate elastic scattered protons. The simulation takes into account the angular distribution of the protons, the energy loss in the PP foil and the energy resolution of the Telescope detectors. The simulation has been performed for several incident energies. The results of these simulations include different configurations of the experimental setup as well as the transmission of the collimators. When the simulated proton spectrum is in agreement with the experimental result (fig. 2(a)), we deduce the neutron 
spectrum (fig. 2(b)), the mean value of the neutron-proton elastic scattering cross section and the efficiency of the proton detector.

Preliminary results for the fission cross section determined in this experiment are displayed in figure 3.

Our results are in agreement with the international evaluations and the data of Knitter et al. ref. [2]. Consequently, our data indicate that the latest results of Laptev et al. ref. [5] over predict this cross section. The overall precision of our data is of $4 \%$ (the statistical uncertainty represents $1 \%$ ). The systematic error includes the uncertainty in the determination of the geometrical efficiency and of the thicknesses of the ${ }^{243} \mathrm{Am}$ target and the polypropylene foil, as well as the uncertainty of the neutron-proton elastic scattering cross section.

\section{Conclusion and perspectives}

We have presented the first measurement of the fission cross section of ${ }^{243} \mathrm{Am}$ in reference to the neutron-proton scattering cross section. This new measurement consolidates the validity of the existing evaluations. In addition, we will use the statistical code of the CENBG to perform our own evaluation of this cross section. In that way, fundamental fission parameters such as fission barrier heights and curvatures will be determined.
These parameters can then be used to infer other neutroninduced cross sections that are much more difficult to measure like capture, inelastic and ( $n, 2 n)$ cross sections.

This work has been supported by the CNRS programme PACE/GEDEPEON and the EURATOM Transnational Acess Programm NUDAME. We thank the Laboratory of Inorganic and Nuclear Chemistry, NY 12201, to make available the ${ }^{243} \mathrm{Am}$ Target and the IPN Orsay for building the high-quality $\Delta \mathrm{E}$ detector of the Si telescope. The authors thank also the VdG accelerator staff of the IRMM at Geel for their great support during the experiment.

\section{References}

1. J.W. Behrens, J.C. Browne, NSE 77, 444 (1981).

2. A.A. Goverdovsky et al., Proc. Int. Conf. Nuclear Data for Basic and Applied Science, Santa Fe, New Mexico, May 13-17, 1985.

3. H.H. Knitter et al., Nucl. Sci. Eng. 99, 1 (1988).

4. P.A. Seeger, Fission Cross Sections from Pommard, LA-4220, 138, Los Alamos National Laboratory (1970).

5. A.B. Laptev et al., Nucl. Phys. A 734, E45 (2004).

6. P. Talou et al., Nucl. Sci. Eng. 155, 84 (2007).

7. J.C. Hopkins, G. Breit, Nucl. Data Tables A 9, 137 (1971).

8. D. Carlson (private communication). 